\title{
Linking Performance with Particle Configuration on Bimetallic Pt/Co/MWCNT Catalysts for Aqueous Phase Reforming by Aberration Corrected STEM coupled with EELS
}

\author{
Cem Akatay ${ }^{1}$, Paul Dietrich ${ }^{2}$, Fred Sollberger ${ }^{2}$, Jeffrey T. Miller $^{3}$, W. Nicholas Delgass ${ }^{2}$, Fabio Ribeiro $^{2}$ \\ and Eric A. Stach \\ 1. School of Materials Engineering and Birck Nanotechnology Center, Purdue University, West \\ Lafayette, Indiana, USA (Currently at UOP LLC, A Honeywell Company) \\ 2. Forney Hall of Chemical Engineering, Purdue University, West Lafayette, Indiana, USA \\ 3. Argonne National Laboratory, Argonne, Illinois 60439, USA \\ 4. Center for Functional Nanomaterials, Brookhaven National Laboratory, Upton, New York 11973, \\ USA
}

Nanoparticles have a higher fraction of atoms exposed to surface and thus exhibit different physical and chemical characteristics than bulk materials. Materials that are immiscible in bulk can form bimetallic nano-species as the heat of formation for alloy nanoparticles is significantly lower than bulk [1]. Bimetallic nanoparticles can have different configurations depending how the constituent elements distribute themselves within the structure. For catalysis, it is of great interest to link the structural characteristics to the observed catalytic properties. Such an understanding can pave the way for the development of catalysts with tailored activity, selectivity and better stability. In this study, the focus will be on the characterization of Pt-Co catalysts supported on multi-wall carbon nanotubes (MWCNT) and on developing an understanding of how particle configuration affects the kinetics of the aqueous phase reforming (APR) of glycerol.

A series of $\mathrm{Pt} / \mathrm{Co} / \mathrm{MWCNT}$ catalysts with different $\mathrm{Pt}$ :Co ratio are prepared to investigate the effect of Co addition on the Pt catalysts supported on multiwall carbon nanotubes. The structure of the catalyst is characterized by aberration corrected scanning transmission electron microscopy coupled with electron energy loss spectroscopy (STEM-EELS). Operando and ex-situ X-ray absorption spectroscopy (XAS) is used to investigate the chemical state and coordination of the Pt and Co species before and during the reaction. The glycerol conversion is promoted by the addition of the Co without compromising selectivity towards hydrogen. The glycerol conversion normalized by the number of sites measured by CO chemisorption, glycerol conversion site time yield (STY), has increased by four times compared to the STY of the cobalt-free sample. Structural characterization revealed a non-uniform structure where different particle morphologies co-exist: Pt only particles, Pt-shell/Co-core particles, as well as well-mixed Pt-Co alloys (Figure 1). Even though, particles with different configurations exhibited same FCC crystal structure (Figure 2), the quantification of different particle morphologies suggested that the promotion benefits from the bimetallic entities (Figure 3).

We acknowledge support from the Institute for Atom-efficient Chemical Transformations (IACT), an Energy Frontier Research Center funded by the U.S. Department of Energy, Office of Science, Office of Basic Energy Sciences. E.S. acknowledges additional support from the Center for Functional Nanomaterials, Brookhaven National Laboratory, which is supported by the U.S. Department of Energy, Office of Basic Energy Sciences, under Contract No. DE-AC02-98CH10886.

Research carried out in part at the Center for Functional Nanomaterials, Brookhaven National Contract No. DE-AC02-98CH10886 
[1] Xiao, et al, The European Physical Journal B (2007), 54
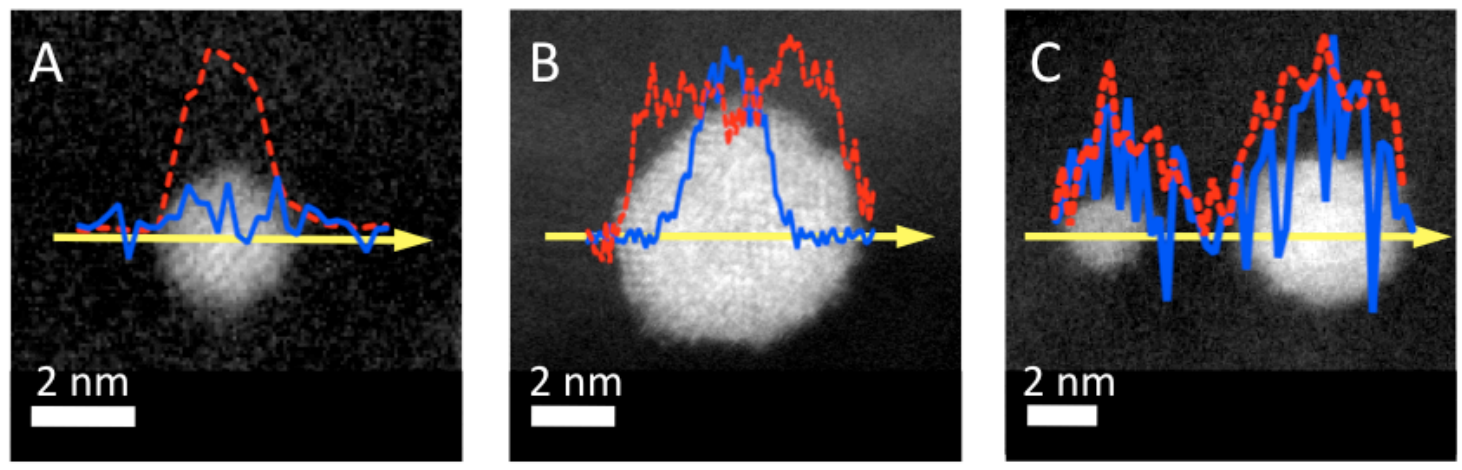

Figure 1. HAADF-STEM micrographs along with STEM-EELS elemental signal profiles for $\mathrm{Pt}$ (red-dashed line) and Co (blue-solid line) feature different particle configurations (A) Pt only, (B) Ptshell/Co-core, (C) Well mixed Pt-Co alloy)
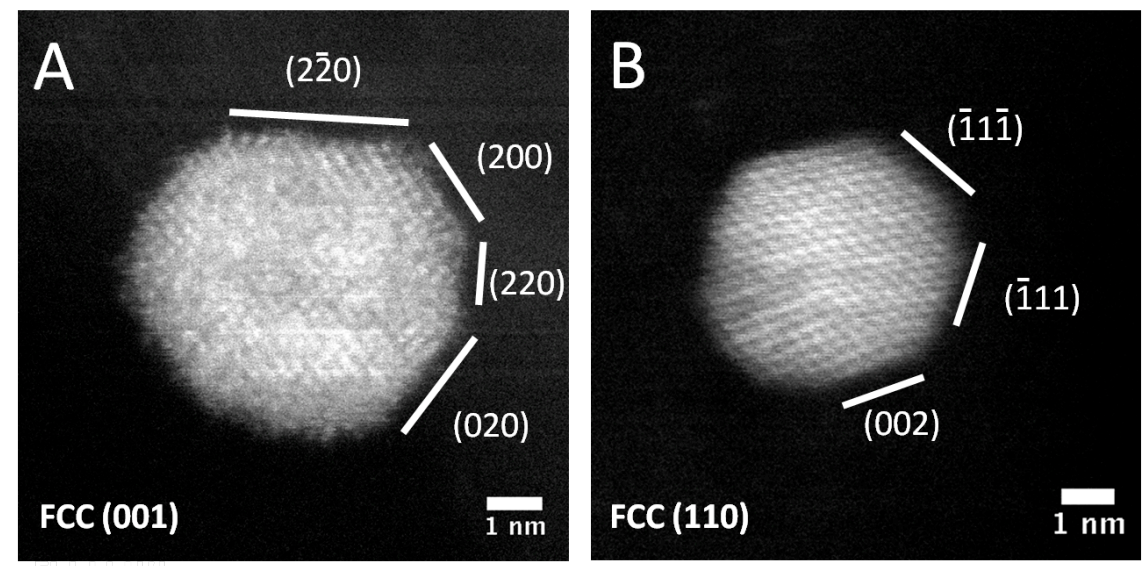

Figure 2. High resolution HAADF-STEM micrographs where surface termination planes are as marked. Both particles are of FCC structure. (A) Pt shell/Co core particle viewed at (001), (B) Well mixed PtCo alloyed viewed at (110)

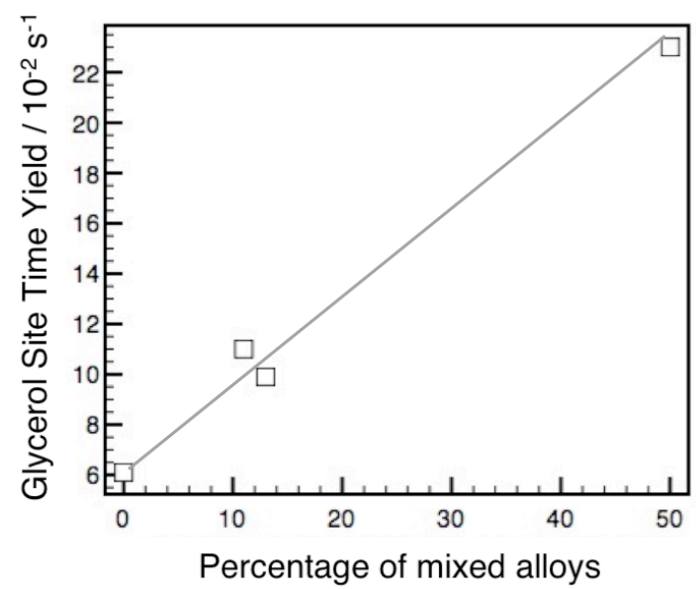

Figure 3. Glycerol conversion site time yield versus fraction of well mixed PtCo alloys 